\title{
Asymptotic safety and conformal standard model
}

\author{
Frederic Grabowski, ${ }^{1}$ Jan H. Kwapisz, ${ }^{2,}$ and Krzysztof A. Meissner ${ }^{2}$ \\ ${ }^{1}$ Faculty of Mathematics, Informatics and Mechanics, University of Warsaw ul. Banacha 2, \\ 02-093 Warsaw, Poland \\ ${ }^{2}$ Faculty of Physics, University of Warsaw ul. Pasteura 5, 02-093 Warsaw, Poland
}

(Received 22 October 2018; published 21 June 2019)

\begin{abstract}
We show that the conformal standard model supplemented with asymptotically safe gravity can be valid up to arbitrarily high energies and give a complete description of particle physics phenomena. We restrict the mass of the second scalar particle to $\sim 300 \mathrm{GeV}$ and the masses of heavy neutrinos to $\sim 340 \mathrm{GeV}$. These predictions can be explicitly tested in the nearby future.
\end{abstract}

DOI: 10.1103/PhysRevD.99.115029

\section{INTRODUCTION}

In recent years there were many extensions of the standard model (SM) proposed to deal with the SM drawbacks like triviality, too weak $C P$ violation for baryogenesis, the hierarchy problem and also the lack of dark matter candidates. Examples of these extensions include grand unification theories [1,2], supersymmetric models [3-5], Higgs portal models [6,7] or the conformal standard model [8-10]. Usually free parameters of these models, like masses of new proposed particles, are known neither theoretically nor experimentally. The possibility of narrowing them down, using some theoretical reasoning, to a small interval would be a huge advantage in the search for new particles and interactions.

One way to restrict the low-energy values of couplings is to include the gravitational corrections to the $\beta$ functions and demand the asymptotic safety condition (AS) on the running of renormalization group equations (RGE) for given initial conditions [11]. These conditions impose bounds on the model free parameters values, which are initial conditions for RGE. Then the model which satisfies them is UV fundamental. However this reasoning is valid only if gravity indeed has a nonperturbative asymptotically safe fixed point. This hypothesis [12] is currently under investigation; however, there are strong indications that it is really so-see for example [13-15]. Much of the work is done using the nonperturbative functional renormalization group methods [16-18]. While the Wetterich equation for flowing action $\Gamma_{k}$ is exact (from which one gets the

\footnotetext{
*Jan.Kwapisz@fuw.edu.pl
}

Published by the American Physical Society under the terms of the Creative Commons Attribution 4.0 International license. Further distribution of this work must maintain attribution to the author(s) and the published article's title, journal citation, and DOI. Funded by SCOAP. effective action $\Gamma=\lim _{k \rightarrow 0} \Gamma_{k}$ ), it is very difficult to deal with. To solve it and hence find the effective action, one has to restrict himself to the finite array of couplings. This, nonperturbative, approach gave the promising results on the mass difference of charged quarks [19] or on the explanation of the top mass [20]. On the other hand, the perturbative approach was used to predict the Higgs mass [11] with astonishing accuracy.

In this article we analyze the conformal standard model (CSM) [8-10], which is of Higgs portal type but has additional structure. This model extends the SM by adding one new complex scalar field with its phase as a dark matter candidate and right-chiral neutrinos. The fundamental assumption which underlies this model and other similar models is that there is no new physics between the weak scale and the Planck scale. This means that for example the masses of heavy neutrinos or vacuum expectation value of new scalars should be of the order of $1 \mathrm{TeV}$. Indeed the observational abbreviations from the standard model such as neutrino masses and oscillations, dark matter and dark energy, baryon asymmetry of the Universe and inflation can be understood without introducing an intermediate new scale; see for example [21,22]. The introduction of the gravitational contributions to the matter beta functions makes all the matter couplings go to a noninteracting fixed point at roughly the Planck scale; hence, at least theoretically it is unnecessary to introduce the new degrees of freedom at some intermediate scale (see [23]) in order to make the standard model a conformal field theory at high energies. Moreover the Large Hadron Collider (LHC) has not detected any discrepancies from the standard model, with no signs of supersymmetry. This is why the Higgs portal models and conformal standard model attract a lot of attention as they can deal, in principle, with the drawbacks of the standard model without changing its structure deeply and adding only a few particles and interactions to the SM. Such models do not possess any 
higher-dimension operators in the Lagrangian, since they are the negative dimensional operators. In the functional renormalization group they should be taken into account. Moreover they can affect the Higgs sector; however, none of these effects have been confirmed yet (see for example [24-26]). Hence in our article we deal (we truncate only to the renormalizable operators) only with those matter operators which are in the Lagrangian of the conformal standard model.

By taking into account the gravitational corrections to the beta functions and using the AS conditions (and assuming that the Weinberg hypothesis holds) we are able to calculate the allowed range of Higgs and the second scalar coupling parameters such that the CSM can be a UV complete theory. By taking into account the experimental LHC data and the model restrictions for the values of free parameters we are able to predict the allowed second scalar mass. We can also narrow down masses of rightchiral neutrinos. One should also mention that there are some studies on models with asymptotically safe behavior without taking into account gravitational corrections [27-30].

\section{MODEL AND METHOD}

\section{A. Higgs portal models and conformal standard model}

In this paragraph we briefly introduce the conformal standard model. The CSM Lagrangian [10] is given by

$$
\mathcal{L}=\mathcal{L}_{\text {kin }}+\mathcal{L}_{Y}-V
$$

with the following kinetic terms:

$$
\begin{aligned}
\mathcal{L}_{\text {kin }}= & \mathcal{L}_{\text {kin }}^{\mathrm{SM}}+\left(D_{\mu} H\right)^{\dagger}\left(D^{\mu} H\right)+\left(\partial_{\mu} \phi^{*} \partial^{\mu} \phi\right) \\
& +i \bar{N}_{\dot{\alpha}}^{j} \bar{\sigma}^{\mu \dot{\alpha} \beta} \partial_{\mu} N_{\beta}^{j},
\end{aligned}
$$

where $H$ is the SM scalar $S U(2)$ doublet. The $\phi(x)$ is a gauge sterile complex scalar field carrying the lepton number and couples only to gauge singlet neutrinos $N_{\alpha}^{i}$. The $\mathcal{L}_{\text {kin }}^{\mathrm{SM}}$ contains all the nonscalar standard model degrees of freedom and can be found in any quantum field theory textbooks; see for example [31,32]. The potential reads as

$$
\begin{aligned}
V(H, \phi)= & -m_{1}^{2} H^{\dagger} H-m_{2}^{2} \phi^{\star} \phi+\lambda_{1}\left(H^{\dagger} H\right)^{2} \\
& +\lambda_{2}\left(\phi^{\star} \phi\right)^{2}+2 \lambda_{3}\left(H^{\dagger} H\right) \phi^{\star} \phi .
\end{aligned}
$$

For the potential (3), if the vacuum expectation values are

$$
\sqrt{2}\left\langle H_{i}\right\rangle=v_{H} \delta_{i 2}, \quad \sqrt{2}\langle\phi\rangle=v_{\phi},
$$

then the tree-level mass parameters are

$$
\begin{aligned}
& m_{1}^{2}=\lambda_{1} v_{H}^{2}+\lambda_{3} v_{\phi}^{2}, \\
& m_{2}^{2}=\lambda_{3} v_{H}^{2}+\lambda_{2} v_{\phi}^{2} .
\end{aligned}
$$

Hence the model possesses two particles of masses: $m_{1}$ and $m_{2}$, where $m_{1}$ is identified with the Higgs particle mass. The potential is bounded from below if

$$
\lambda_{1}>0, \quad \lambda_{2}>0, \quad \lambda_{3}>-\sqrt{\lambda_{2} \lambda_{1}} .
$$

If, in addition to (7) the $\lambda_{3}<\sqrt{\lambda_{1} \lambda_{2}}$ holds, then (4) is the global minimum of $V$. For given $\lambda_{1}, \lambda_{2}, \lambda_{3}$ one can calculate $v_{\phi}$ and $m_{2}$ at tree level using (5) and at loop level by changing the renormalization schemes, because $v_{H}$ is known experimentally and the Higgs mass resulting from AS can be calculated, when we take $\lambda_{3}=0$. With $\lambda_{0}=\frac{1}{2} \frac{m_{1}^{2}}{v_{H}^{2}} \approx 0.13$, one can parametrize the deviation from $\mathrm{SM}$ as

$$
\tan \beta=\frac{\lambda_{0}-\lambda_{1}}{\lambda_{3}} \frac{v_{H}}{v_{\phi}} .
$$

We assume that its value is restricted by $|\tan \beta|<0.35$ which is the limit allowed by the present LHC data [10]. This constraint will be used to narrow down the possible values of $m_{2}$. In the conformal standard model the coupling constants $Y_{i j}^{M}$ and $Y_{i j}^{\nu}$ are introduced, which are responsible for interactions of right-chiral neutrinos:

$\mathcal{L}_{Y}=\frac{1}{2} Y_{j i}^{M} \phi N^{j \alpha} N_{\alpha}^{i}+Y_{j i}^{\nu} N^{j \alpha} H^{\top} \epsilon L_{\alpha}^{i}+\mathcal{L}_{Y}^{\mathrm{SM}}+$ H.c.,

where $\mathcal{L}_{Y}^{\mathrm{SM}}$ is Yukawa part of the standard model Lagrangian part and $\epsilon$ is the antisymmetric $S U(2)_{L}$ metric. Following [10] we assume the degeneracy of Yukawa couplings $Y_{i j}^{M}=y_{M} \delta_{i j}$, which amplifies the $C P$ violation and makes the resonant leptogenesis scenario possible; see $[9,10,33]$ for details. The masses of right-chiral neutrinos are given by

$$
M_{N}=y_{M} v_{\phi} / \sqrt{2}
$$

for leptogenesis to take place, one requires $M_{N}>m_{2}$, so that the heavy neutrinos can decay. Moreover the conformal standard model introduces a phase of the second scalar particle, called minoron, which can be a potential dark matter candidate, with mass $v^{4} / M_{P}^{2}$ originating from quantum gravity effects, where $v$ is some new parameter with $v \sim v_{\phi}$. The CSM beta functions in the $\overline{\text { MS }}$ scheme, where $\hat{\beta}_{\mathrm{CSM}}=16 \pi^{2} \beta_{\mathrm{CSM}}$, are given by [10] 


$$
\begin{aligned}
\hat{\beta}_{g_{1}}= & \frac{41}{6} g_{1}^{3}, \quad \hat{\beta}_{g_{2}}=-\frac{19}{6} g_{2}^{3}, \quad \hat{\beta}_{g_{3}}=-7 g_{3}^{3}, \\
\hat{\beta}_{y_{t}}= & y_{t}\left(\frac{9}{2} y_{t}^{2}-8 g_{3}^{2}-\frac{9}{4} g_{2}^{2}-\frac{17}{12} g_{1}^{2}\right), \\
\hat{\beta}_{\lambda_{1}}= & 24 \lambda_{1}^{2}+4 \lambda_{3}^{2}-3 \lambda_{1}\left(3 g_{2}^{2}+g_{1}^{2}-4 y_{t}^{2}\right) \\
& +\frac{9}{8} g_{2}^{4}+\frac{3}{4} g_{2}^{2} g_{1}^{2}+\frac{3}{8} g_{1}^{4}-6 y_{t}^{4}, \\
\hat{\beta}_{\lambda_{2}}= & \left(20 \lambda_{2}^{2}+8 \lambda_{3}^{2}+6 \lambda_{2} y_{M}^{2}-3 y_{M}^{4}\right), \\
\hat{\beta}_{\lambda_{3}}= & \frac{1}{2} \lambda_{3}\left[24 \lambda_{1}+16 \lambda_{2}+16 \lambda_{3}-\left(9 g_{2}^{2}+3 g_{1}^{2}\right)+6 y_{M}^{2}+12 y_{t}^{2}\right], \\
\hat{\beta}_{y_{M}}= & \frac{5}{2} y_{M}^{3},
\end{aligned}
$$

where $g_{1}, g_{2}$, and $g_{3}$ are $U(1), S U(2)$, and $S U(3)$ standard model gauge couplings, respectively, and $y_{t}$ is the top Yukawa coupling. Following [10] we do not take into account the running of $Y^{\nu}$. If one takes $y_{M}=0$, then after the redefinitions of the couplings the CSM beta functions reduce to the Higgs portal ones with [6,34-38].

\section{B. Asymptotic safety and gravitational corrections}

Weinbergs' notion of AS [12] can be summarized by his quote: "A theory is said to be asymptotically safe if the essential coupling parameters approach a fixed point as the momentum scale of their renormalization point goes to infinity." Let us assume that $\mathbf{g}$ is a set of all the couplings of a theory and let $\left.\mathbf{g}\right|_{\mu_{0}}$ be a given a set of initial conditions at some momentum scale $\mu_{0}$. In our case we take $\mu_{0}=173.34 \mathrm{GeV}$; see $[39,40]$. These initial conditions together with the set of equations for running of couplings,

$$
\beta_{i}(\mathbf{g}(\mu))=\mu \frac{\partial}{\partial \mu} g_{i}(\mu)
$$

describe completely and uniquely the behavior of a physical theory. If for some $g_{i}$ we have $\beta_{i}\left(\mathbf{g}^{*}\right)=0$, then we call this $\mathbf{g}^{*}$ a fixed point of the $i$ th equation. The stable fixed points are called attractors, and the unstable one are called repellers. If $\lim _{\mu \rightarrow+\infty} \mathbf{g}^{*} \equiv 0$ for all the couplings $g_{i}$, we call such a point a Gaussian fixed point. Theories where the couplings possess a Gaussian fixed at UV scales are called asymptotically free. Otherwise, when $g^{*} \neq 0$ we call such a fixed point non-Gaussian and interacting, and such theories are called asymptotically safe. If the equation for running of the coupling $g_{i}$ possesses an unstable fixed points at UV scale, then there is only one low-energy initial condition $\left.g_{i}\right|_{\mu_{0}}$ per repeller such that the theory is fundamental up to the UV scale.

On the other hand, gravity cannot be perturbatively quantized. Nevertheless one can utilize the effective field theory approach for energies below the Planck scale to determine predictions of quantum gravity. In particular the standard model (and its extensions) $\beta$ functions are modified with the gravitational corrections at high energies $[11,13,39,41-45]$ :

$$
\beta_{i}(\mathbf{g})=\beta_{i}^{\text {matter }}(\mathbf{g})+\beta_{i}^{\text {grav }}(\mathbf{g}, \mu) .
$$

The gravitational contributions to the beta functions acquire the general form for all the matter couplings $[11,13,39,41,45]$ :

$$
\beta_{i}^{\text {grav }}(\mathbf{g}, \mu)=\frac{a_{i}}{8 \pi} \frac{\mu^{2}}{M_{P}^{2}+\xi_{0} \mu^{2}} g_{i}
$$

due to the universal character of gravitational interactions. $M_{P}=\left(8 \pi G_{N}\right)^{-1 / 2}=2.4 \times 10^{18} \mathrm{GeV}$ is the low-energy Planck mass. $\xi_{0}$ is some dimensionless constant. Based on the results of a functional renormalization group investigation of pure gravity [11,44,46,47] its value is taken as $\xi_{0}=0.024$. The $a_{i}$ are dimensionless constants and can be calculated for a given coupling $g_{i}$. According to $[39,41,42]$ one have $a_{g_{1}}=a_{g_{2}}=a_{g_{3}}=-1$ and $a_{y_{t}}=-0.5$ at the one-loop level. Since $y_{M}$ is a gauge coupling we assume that also $a_{y_{M}}=-1$. The value $a_{\lambda_{1}}=+3$ is based on $[11,39,47,48]$. For simplicity we assume that all of the $a_{\lambda_{i}}$ have the same absolute value, which can be supported by the calculations of the $a_{\lambda}$ parameters done for the Higgs portal models [49]. However this calculation does not take into account fermions or higher-order operators for gravity. For example the theory with the $R^{2}$ term in the gravity Lagrangian can be identified with the scalar tensor theory of gravity [50,51] which is used to describe inflation, and this term has negative $a_{R^{2}}$ [52] and so does the scalar. So we investigate all the possibilities in our article. To show that the absolute value of $a_{\lambda_{2}}, a_{\lambda_{3}}$ is not an important factor when concerning the possible masses we have scanned over several values lying in the interval $\left|a_{\lambda_{2}}\right|,\left|a_{\lambda_{3}}\right| \in[1,3]$. Its change affects the allowed mass very weakly, of the order of $\pm 2 \mathrm{GeV}$; this is because the gravitational corrections are heavily suppressed below the Planck scale. Indeed, the sign of $a_{i}$ is much more important than its exact value because the positive sign corresponds to the unstable Gaussian fixed point while the negative to the stable one. In result we investigate the four possibilities: $a_{\lambda_{2}}, a_{\lambda_{3}}= \pm 3$.

The asymptotic safety assumption for quantum gravity allows us to treat (extensions of) the standard model as fundamental (UV complete) only under the condition that the running of coupling constants does not possess any pathological behavior up to the Planck scale. It imposes two conditions $[10,11]$. We will call them AS conditions. Firstly, there should be no Landau poles up to the Planck scale. Secondly, the electroweak vacuum should be stable for all scales:

$\lambda_{1}(\mu)>0, \quad \lambda_{2}(\mu)>0, \quad \lambda_{3}(\mu)>-\sqrt{\lambda_{2}(\mu) \lambda_{1}(\mu)}$. 
The second condition comes from the assumption that there is essentially no new physics between the electroweak scale and Planck scale, despite the one described by the conformal standard model. Obviously at the Planck scale all the matter couplings go to zero, and hence all the beta functions go to zero. The next paragraph is dedicated to the calculation of the lambda couplings $\left(\lambda_{1}, \lambda_{2}, \lambda_{3}, y_{M}\right)$ satisfying these conditions.

\section{CALCULATION OF LAMBDA COUPLINGS}

In this paragraph we calculate the set of allowed lambda couplings satisfying the asymptotic safety conditions. If not specified otherwise, the value of a coupling (for example on the plots below) means its value at $\mu_{0}=173.34$. In the lowenergy regime the graviton loops can be neglected $[11,53,54]$ and they become important near the Planck scale and manifest in the form of the gravitational corrections to the $\beta$ functions. The gauge and Yukawa couplings renormalization group equations dynamics is not affected by the running of $\lambda_{i}$ and $y_{M}$. The low-energy values at $\mu_{0}=173.34$ are taken as [39] $g_{1}\left(\mu_{0}\right)=0.35940$, $g_{2}\left(\mu_{0}\right)=0.64754, g_{3}\left(\mu_{0}\right)=1.1888$ and $y_{t}\left(\mu_{0}\right)=0.95113$. Then the evolution of these couplings with energy is obtained. The running of $y_{M}$ is also independent from all other couplings. So far the experimental value for $y_{M}$ is unknown, so to obtain the allowed $\lambda_{i}\left(y_{M}\right)$ one has to scan over all possible values of $y_{M}$. Using the AS conditions we have calculated the allowed interval of $y_{M}$ for $a_{y_{M}}=-1$ as $y_{M} \in[0.0,0.925]$, and for $a_{y_{M}}=+1$ we have $y_{M}=0.0$. We plug the evolution of the gauge and Yukawa couplings and each allowed $y_{M}$ into coupled equations for $\lambda_{1}, \lambda_{2}, \lambda_{3}$. Moreover, the magnitudes of $a_{i}$ coefficients are such that the theory becomes asymptotically free near the Planck scale; see [11] for further details, which justifies the use of perturbation approach. Furthermore we expect that if the cosmological constant runs, then it does not affect the matter couplings below the Planck scale. This reasoning is supported by the fact that both the Higgs mass [11] and the mass difference between the top and bottom quark were accurately predicted [55] without taking into account the running of the cosmological constant. Furthermore in the case of the unimodular gravity [56], which is equivalent to the Einstein theory at the classical level, the cosmological constant is not a dynamical degree of freedom; hence, it does not run at all. These arguments suggest that we do not take the cosmological constant running into account. As a result we are looking for the sets of initial values $y_{M}, \lambda_{i}$ such that they will all drop to zero near the Planck scale.

\section{A. Coefficients: $a_{\lambda_{3}}=-3, a_{\lambda_{2}}=-3$}

We start with the most general case, when $a_{\lambda_{2}}=a_{\lambda_{3}}=-3$. Since for each possible combination of $\lambda_{2}, \lambda_{3}, y_{M}$ only one $\lambda_{1}$ is allowed, then we consider a set $\mathfrak{M}$ of allowed $\lambda_{2}, \lambda_{3}, y_{M}\left(\lambda_{2}\right.$ and $\lambda_{3}$ depend mutually on each

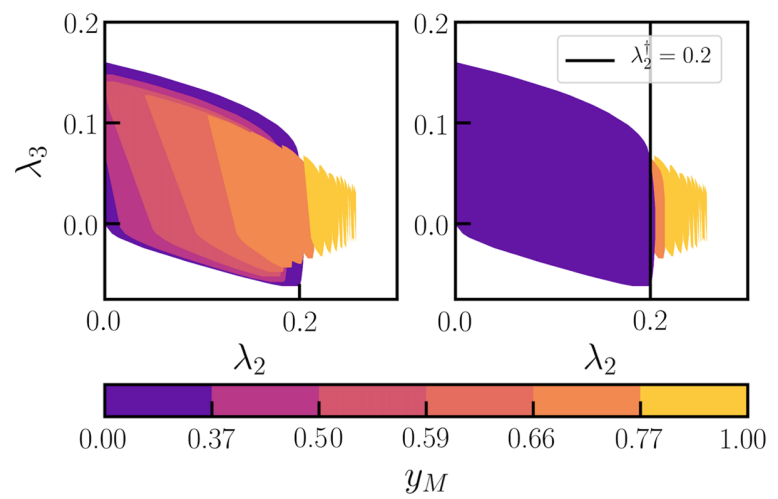

FIG. 1. Maximal (left) and minimal (right) $y_{M}\left(\lambda_{3}, \lambda_{2}\right)$, $a_{\lambda_{2}}=-3, a_{\lambda_{3}}=-3$.

other and on $\left.y_{M}\right)$, and there is a $\lambda_{1}\left(\lambda_{2}, \lambda_{3}, y_{M}\right)$ assigned to each of the points of this set. This set looks roughly like a sea wave, where $y_{M}$ is the height. In Fig. 1 we show the surfaces of maximal and minimal possible $y_{M}$ all the points in between are also in the set $\mathfrak{M}$.

In the right figure there is a special line $\lambda_{2}^{\dagger}=0.2$. Namely, for the region where $\lambda_{2}<\lambda_{2}^{\dagger}$ the minimal $y_{M}$ is 0.0 , while when $\lambda_{2}>\lambda_{2}^{\dagger}$ the minimal and maximal values are very close to each other: $y_{M}(\max )-y_{M}(\min ) \sim 0.01$. This behavior can be explained by the observation that $\lambda_{2}>$ $\lambda_{2}^{\dagger}$ requires $y_{M}>0.70$ to keep $\hat{\beta}_{\lambda_{2}}$ small enough throughout the evolution. As we have checked in all other cases the sets of allowed couplings form a $1 \mathrm{D}$ or $2 \mathrm{D}$ subsets of $\mathfrak{M}$.

The $\lambda_{1}$ renormalization group equation is affected directly only by $\lambda_{3}$; however, the running of $\lambda_{3}$ depends on $\lambda_{2}$ and $y_{M}$. In Fig. 2 we show the $\lambda_{1}$ dependence on other couplings for three chosen $y_{M}$ as an example.

\section{B. Coefficients: $a_{\lambda_{3}}=-3, a_{\lambda_{2}}=+3$}

In this case there is one allowed $\lambda_{1}$ and $\lambda_{2}$ per set of $\lambda_{3}$ and $y_{M}$ satisfying the AS conditions. In Fig. 3 we present this dependence. As we can see there is much less spread in possible values of $\lambda_{1}$ than $\lambda_{2}$. This is because all the gauge coupling initial values are fixed for $\lambda_{1}$, which is not the case for $\lambda_{2}$.

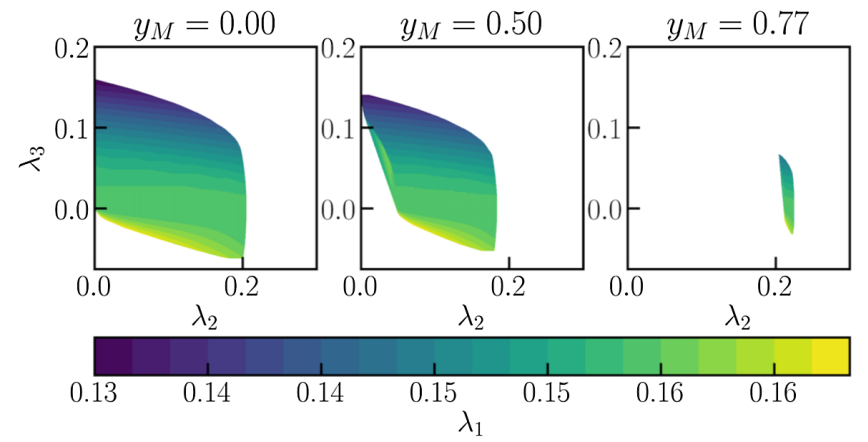

FIG. 2. Values of $\lambda_{1}$ for $y_{M}=0.0$ (left), $y_{M}=0.5$ (mid), and $y_{M}=0.77$ (right). 

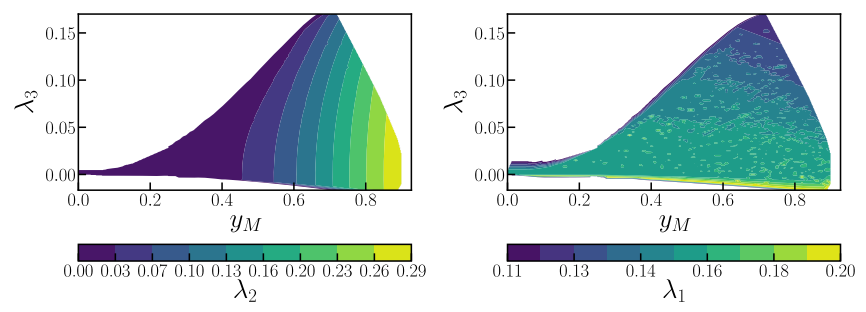

FIG. 3. $\lambda_{1}$ dependence on $\lambda_{3}$ and $y_{M}, a_{\lambda_{2}}=+3, a_{\lambda_{3}}=-3$.

For this case the domain of allowed $\lambda_{3}$ and $y_{M}$ is smaller than for $\mathfrak{M}$. However for this domain we have $\lambda_{2}\left(a_{\lambda_{3}}=+3, \lambda_{3}, y_{M}\right)=\min _{\lambda_{2}}\left(\lambda_{2}\left(a_{\lambda_{2}}=-3, \lambda_{3}, y_{M}\right)\right\}$.

\section{Coefficients: $a_{\lambda_{3}}=+3, a_{\lambda_{2}}= \pm \mathbf{3}$}

In this case, we found that only $\lambda_{3}\left(\mu_{0}\right)=0$ satisfies the AS conditions; hence, $\lambda_{3}$ is zero at all energy scales. Then the $\phi$ sector is decoupled from the rest of standard model, which makes it a scalar dark matter candidate, like in [49]. The numerical solution for $\lambda_{1}$ gives $\lambda_{1}=0.1537$, which agrees the standard model predictions from asymptotic safety; see [11,39]. The allowed region for $\lambda_{2}$ is determined by the AS conditions for this coupling and depends heavily on $y_{M}$.

We checked that the allowed value for $\lambda_{2}$ shown in Fig. 4 is the subset of $\mathfrak{M}$ with the condition $\lambda_{3}=0.0$. We have also calculated that $\lambda_{2}\left(a_{\lambda_{2}}=+3, y_{M}\right)$ mimics the lower bound for $\lambda_{2}\left(a_{\lambda_{2}}=-3, y_{M}\right)$. Moreover this bound is the same as for the running of $\lambda_{2}$ without gravitational corrections.

\section{THE SECOND SCALAR MASS}

In this paragraph we calculate the $v_{\phi}, m_{2}$ and $M_{N}$. For the decoupled (standard model) case one obtains $\lambda_{1}=$ 0.1537 ; hence, the Higgs mass is given by

$$
m_{1}=\sqrt{2 \lambda_{1} v_{H}^{2}} \approx 136 \mathrm{GeV}
$$

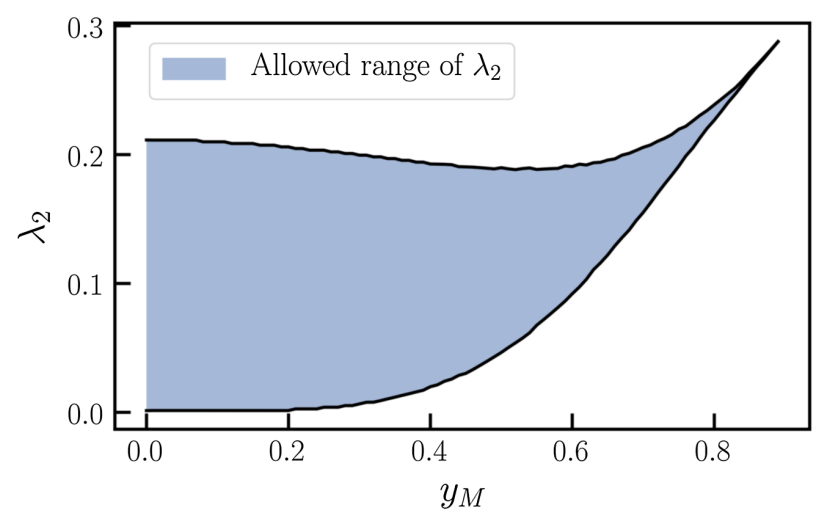

FIG. 4. Allowed range of $\lambda_{2}$ coupling, $a_{\lambda_{2}}=-3, a_{\lambda_{3}}=+3$.
TABLE I. Arrays of allowed masses.

\begin{tabular}{cccccc}
\hline \hline$a_{\lambda_{2}}$ & $2 m_{1}<m_{2}$ & $y_{M}$ & $m_{2}[\mathrm{GeV}]$ & $v_{\phi}[\mathrm{GeV}]$ & $M_{N}[\mathrm{GeV}]$ \\
\hline+3 & Yes & 0.84 & 275 & 538 & 319 \\
+3 & Yes & 0.85 & 296 & 574 & 345 \\
-3 & Yes & $0.77_{-0.06}^{+0.07}$ & $300_{-28}^{+28}$ & $586_{-46}^{+60}$ & $342_{-41}^{+41}$ \\
-3 & No & 0.00 & $160_{-100}^{+103}$ & $300_{-15}^{+275}$ & $\mathrm{NA}$ \\
\hline \hline
\end{tabular}

where the $\lambda_{1}$ is calculated from the AS requirements and is in the $\overline{\mathrm{MS}}$ scheme. So one can see that the effects regarding the renormalization of mass and the ones concerning the changing of the renormalization scheme (from $\overline{\mathrm{MS}}$ to the physical one) have the contribution of the order of $10 \mathrm{GeV}$ to the masses calculated at the tree-level relations (without renormalization of mass), which was demonstrated in $[11,39]$. Hence due to other much bigger sources of uncertainties like the value of $y_{M}$, we assume that the CSM tree-level relations (5), (6) hold. This assumption restricts $\lambda_{3}$ to be non-negative; otherwise, one would obtain negative values for $v_{\phi}^{2}$ sufficient for the calculations of the allowed $\lambda$ couplings. By comparing the one-loop [39] and two-loop calculation [11] of $\lambda_{1}$, where the outcome is almost identical, we conclude that higher than the first nontrivial order is sufficient for our purpose. In order to calculate $v_{\phi}$ and $m_{2}$ at tree level we take $v_{H}=246 \mathrm{GeV}$, and then we solve Eqs. (5) and (6) with $m_{1}=136 \mathrm{GeV}$. Obviously we are able to predict the $m_{2}$ and $v_{\phi}$ only in the case when the second scalar sector is coupled to the SM sector. Hence, we restrict only to the case when $\lambda_{3} \neq 0$. For small $\lambda_{3}$ tiny changes in $\lambda_{1}$ result in enormous changes in $v_{\phi}$, so we treat $\lambda_{3}<0.01$ as a decoupled case. Our claim that $\lambda_{3}$ should be large enough can also be justified with the LHC condition $|\tan \beta|<0.35$, because small $\lambda_{3}$ results in large $\beta$. In our analysis we have excluded all the sets of parameters not satisfying the LHC and the global stability conditions [at $\left.\mu_{0}: \sqrt{\lambda_{1}\left(\mu_{0}\right) \lambda_{2}\left(\mu_{0}\right)}>\lambda_{3}\left(\mu_{0}\right)\right]$. After doing that we have two separate cases: $a_{\lambda_{2}}=+3$ and $a_{\lambda_{2}}=-3$. Moreover if the second scalar particle is unstable (following the CSM [10]), then at the tree level we have $2 m_{1}<m_{2}$. Also the $y_{M}=0.0$ situation is an interesting situation, when the CSM reduces to the Higgs portal case. The masses of right-handed neutrinos can also be calculated, $M_{N}=y_{M} v_{\phi} / \sqrt{2}$ in the case when $y_{M} \neq 0$. Below we illustrate these combinations of possible conditions.

In Table I, in the second row "yes" means that we have taken into account this condition, while "no" means the opposite. As we can see for $y_{M}=0.0$ the second scalar mass is $m_{2}$ is smaller than $2 m_{1}$ making this particle stable. In the conformal standard model case the right-chiral neutrinos turns out to be unstable $\left(m_{2}<y_{M} v_{\phi} / \sqrt{2}\right.$ holds for each of the sets of parameters) making the leptogenesis scenario possible. Furthermore if we assume that $v=v_{\phi}$, 
then the mass of the minoron is given by $v_{\phi}^{2} / M_{P} \approx 10^{-4} \mathrm{eV}$, which is of the same order as estimated in [10].

We have also analyzed the running of the $\beta$ functions for $m_{2}$ and $m_{1}$, where we took $a_{m_{i}}=-1$. It gives no new bounds on $m_{2}$ and lambda couplings. This result is in close relation with scenario B analyzed in [49]; however in our case the $\phi$ particle is not decoupled from the SM. We have also taken into account the restrictions from quadratic divergence cancellation, which can be achieved if softly broken conformal symmetry (SBCS) [53] requirements are satisfied (these requirements in the CSM are not essentially changed even after adding gravitational corrections [54]). Apparently it gives no new restrictions for the parameters additional to the asymptotic safety conditions. Moreover the SBCS requirements are satisfied at $E=M_{P}$ with all the couplings becoming asymptotically free, which was suggested in [53].

\section{CONCLUSIONS}

Our investigation shows that the conformal standard model has a set of parameters compatible with the asymptotic safety conditions, so supplemented with gravitational corrections it can be a UV fundamental theory and [10] "allows for a comprehensive treatment of all outstanding problems of particle physics." Moreover, the CSM can be slightly modified to incorporate an inflation scenario [38,57], which agrees with 2013 Planck data analysis [58-60]. Inflation can also take place due to the asymptotically safe scenario [61].

The restrictions on the couplings and masses derived in this article allow us to make theoretical predictions for free parameters of the models extending the standard model. The lambda couplings can be directly measured (or explicitly calculated from masses of the new scalar particles) in the LHC or indirectly measured in cosmological observations. In particular our investigation supports the claim [62] that the excess of events with four charged leptons at $E \sim 325 \mathrm{GeV}$ seen by the CDF [63] and CMS [64] Collaborations can be identified with a detection of a new "sterile" scalar particle proposed by the conformal standard model. On the other hand, the compatibility of the LHC run 1 data with the heavy scalar hypothesis was investigated in $[65,66]$. The hypothetical heavy boson mass is measured to be around $272 \mathrm{GeV}$ (in the $270-320 \mathrm{GeV}$ range). Hence we would like to emphasize that this experimental analysis agrees with the theoretical range provided by our calculations and with the claim discussed in [62] at least up to the order of several GeV. The fact that $m_{2}>2 m_{1}$ implies the $y_{M} \neq 0.0$ underlies the role of righthanded neutrinos not only in context of smallness of neutrino masses, but also in the case of asymptotically safe beyond-SM physics, which makes the conformal standard model unique among the Two-Higgs-doublet model and Higgs portal models.

We hope that our analysis will be helpful in search for new particles at LHC and future colliders and the new scalar particle predicted by this model can be detected in the nearby future.

\section{ACKNOWLEDGMENTS}

We thank Piotr Chankowski for valuable discussions. J.H.K. would like to thank Yukawa Institute for Theoretical Physics for hospitality and support during this work. The PL-Grid Infrastructure is gratefully acknowledged. K. A. M. was partially supported by the Polish National Science Center Grant No. DEC-2017/25/B/ST2/ 00165. J. H. K. was supported by the Polish National Science Centre Grant No. 2018/29/N/ST2/01743.
[1] H. Georgi and S. L. Glashow, Phys. Rev. Lett. 32, 438 (1974).

[2] A. Buras, J. Ellis, M. Gaillard, and D. Nanopoulos, Nucl. Phys. B135, 66 (1978).

[3] S. Dimopoulos, S. Raby, and F. Wilczek, Phys. Rev. D 24, 1681 (1981).

[4] L. Ibáñez and G. Ross, Phys. Lett. 105B, 439 (1981).

[5] S. Dimopoulos and H. Georgi, Nucl. Phys. B193, 150 (1981).

[6] B. Patt and F. Wilczek, arXiv:hep-ph/0605188.

[7] M. Shaposhnikov and I. Tkachev, Phys. Lett. B 639, 414 (2006).

[8] K. A. Meissner and H. Nicolai, Phys. Lett. B 648, 312 (2007).
[9] A. Latosinski, A. Lewandowski, K. A. Meissner, and H. Nicolai, J. High Energy Phys. 10 (2015) 170.

[10] A. Lewandowski, K. A. Meissner, and H. Nicolai, Phys. Rev. D 97, 035024 (2018).

[11] M. Shaposhnikov and C. Wetterich, Phys. Lett. B 683, 196 (2010).

[12] S. Weinberg, in General Relativity: An Einstein Centenary Survey, edited by S. W. Hawking and W. Israel (Cambridge University Press, Cambridge, UK, 1979), pp. 790-831.

[13] A. Eichhorn, Found. Phys. 48, 1407 (2018).

[14] O. Lauscher and M. Reuter, in Quantum Gravity: Mathematical Models and Experimental Bounds, edited by B. Fauser et al. (2005), pp. 293-313, https://inspirehep.net/ search?p=find+eprint+hep-th/0511260. 
[15] A. Salvio and A. Strumia, Eur. Phys. J. C 78, 124 (2018).

[16] C. Wetterich, Phys. Lett. B 301, 90 (1993).

[17] T. R. Morris, Int. J. Mod. Phys. A 9, 2411 (1994).

[18] T. R. Morris, Phys. Lett. B 329, 241 (1994).

[19] A. Eichhorn and A. Held, Phys. Rev. Lett. 121, 151302 (2018).

[20] A. Eichhorn and A. Held, Phys. Lett. B 777, 217 (2018).

[21] A. Boyarsky, O. Ruchayskiy, and M. Shaposhnikov, Annu. Rev. Nucl. Part. Sci. 59, 191 (2009).

[22] M. Shaposhnikov, in Astroparticle Physics: Current Issues, 2007 (APCI07) Budapest, Hungary, 2007 (2007).

[23] G. Marques Tavares, M. Schmaltz, and W. Skiba, Phys. Rev. D 89, 015009 (2014).

[24] B. Grzadkowski, M. Iskrzynski, M. Misiak, and J. Rosiek, J. High Energy Phys. 10 (2010) 085.

[25] J. Borchardt, H. Gies, and R. Sondenheimer, Eur. Phys. J. C 76, 472 (2016).

[26] R. Sondenheimer, Eur. Phys. J. C 79, 10 (2019).

[27] M. Badziak and K. Harigaya, Phys. Rev. Lett. 120, 211803 (2018).

[28] G. M. Pelaggi, A. D. Plascencia, A. Salvio, F. Sannino, J. Smirnov, and A. Strumia, Phys. Rev. D 97, 095013 (2018).

[29] R. Mann, J. Meffe, F. Sannino, T. Steele, Z. Wang, and C. Zhang, Phys. Rev. Lett. 119, 261802 (2017).

[30] D. Barducci, M. Fabbrichesi, C. M. Nieto, R. Percacci, and V. Skrinjar, J. High Energy Phys. 11 (2018) 057.

[31] S. Weinberg, The Quantum Theory of Fields (Cambridge University Press, Cambridge, England, 1995), Vol. 1.

[32] S. Pokorski, Gauge Field Theories, 2nd ed., Cambridge Monographs on Mathematical Physics (Cambridge University Press, Cambridge, England, 2000).

[33] A. Pilaftsis and T. E. J. Underwood, Nucl. Phys. B692, 303 (2004).

[34] G. C. Branco, P. M. Ferreira, L. Lavoura, M. N. Rebelo, M. Sher, and J. P. Silva, Phys. Rep. 516, 1 (2012).

[35] J. F. Gunion and H.E. Haber, Phys. Rev. D 67, 075019 (2003).

[36] J. D. Wells, in 39th British Universities Summer School in Theoretical Elementary Particle Physics (BUSSTEPP 2009) Liverpool, United Kingdom, 2009 (2009).

[37] J. O. Gong, H. M. Lee, and S. K. Kang, J. High Energy Phys. 04 (2012) 128.

[38] O. Lebedev and H. M. Lee, Eur. Phys. J. C 71, 1821 (2011),

[39] L. Laulumaa, Higgs mass predicted from the standard model with asymptotically safe gravity, Master's thesis, Jyvaskyla University, 2016.
[40] D. Buttazzo, G. Degrassi, P. P. Giardino, G. F. Giudice, F. Sala, A. Salvio, and A. Strumia, J. High Energy Phys. 12 (2013) 089.

[41] S. P. Robinson and F. Wilczek, Phys. Rev. Lett. 96, 231601 (2006).

[42] O. Zanusso, L. Zambelli, G. P. Vacca, and R. Percacci, Phys. Lett. B 689, 90 (2010).

[43] L. Griguolo and R. Percacci, Phys. Rev. D 52, 5787 (1995).

[44] R. Percacci and D. Perini, Phys. Rev. D 68, 044018 (2003).

[45] A. Eichhorn, Y. Hamada, J. Lumma, and M. Yamada, Phys. Rev. D 97, 086004 (2018).

[46] M. Reuter, Phys. Rev. D 57, 971 (1998).

[47] R. Percacci and D. Perini, Phys. Rev. D 68, 044018 (2003).

[48] G. Narain and R. Percacci, Classical Quantum Gravity 27, 075001 (2010).

[49] A. Eichhorn, Y. Hamada, J. Lumma, and M. Yamada, Phys. Rev. D 97, 086004 (2018).

[50] F. L. Bezrukov and M. Shaposhnikov, Phys. Lett. B 659, 703 (2008).

[51] A. A. Starobinsky, JETP Lett. 30, 682 (1979).

[52] A. Eichhorn, arXiv:1810.07615.

[53] P. H. Chankowski, A. Lewandowski, K. A. Meissner, and H. Nicolai, Mod. Phys. Lett. A 30, 1550006 (2015).

[54] K. A. Meissner, H. Nicolai, and J. Plefka, Phys. Lett. B 791, 62 (2019).

[55] A. Eichhorn and A. Held, Phys. Rev. Lett. 121, 151302 (2018).

[56] A. Eichhorn, Classical Quantum Gravity 30, 115016 (2013).

[57] J. H. Kwapisz and K. A. Meissner, Acta Phys. Pol. B 49, 115 (2018).

[58] P. A. R. Ade et al. (BICEP2 and Planck Collaborations), Phys. Rev. Lett. 114, 101301 (2015).

[59] A. H. Guth, D. I. Kaiser, and Y. Nomura, Phys. Lett. B 733, 112 (2014).

[60] D. I. Kaiser, Fundam. Theor. Phys. 183, 41 (2016).

[61] S. Weinberg, Phys. Rev. D 81, 083535 (2010).

[62] K. A. Meissner and H. Nicolai, Phys. Lett. B 718, 943 (2013).

[63] T. Aaltonen et al. (CDF Collaboration), Phys. Rev. D 85, 012008 (2012).

[64] S. Chatrchyan et al. (CMS Collaboration), Phys. Rev. Lett. 108, 111804 (2012).

[65] S. von Buddenbrock, N. Chakrabarty, A. S. Cornell, D. Kar, M. Kumar, T. Mandal, B. Mellado, B. Mukhopadhyaya, and R. G. Reed, arXiv:1506.00612.

[66] S. von Buddenbrock, N. Chakrabarty, A. S. Cornell, D. Kar, M. Kumar, T. Mandal, B. Mellado, B. Mukhopadhyaya, R. G. Reed, and X. Ruan, Eur. Phys. J. C 76, 580 (2016). 